Faculty of Sciences and Mathematics, University of Niš, Serbia

Available at: http://www.pmf.ni.ac.rs/filomat

Filomat 24:4 (2010), 53-61 DOI: 10.2298/FIL1004053A

\title{
SOME PROPERTIES OF HYPERSPACES OF ČECH CLOSURE SPACES WITH VIETORIS-LIKE TOPOLOGIES
}

\author{
Dimitrije Andrijević, Milena Jelić, Mila Mrševićc*
}

\begin{abstract}
We study some topological properties of hyperspaces of Čech closure spaces endowed with Vietoris-like topologies. Some of these notions were introduced and considered in $[9,10]$ and $[11]$, focussing on selection principles.
\end{abstract}

\section{Introduction}

In the first part of the book Topological Spaces [1] the theory of topological spaces is developed by considering the closure operator which need not be idempotent. We call such an operator a closure operator in the sense of Čech, or a Čech closure operator. In $[2,3,4]$ different types of continuous-like functions between topological spaces were considered and topologies on sets of these function investigated. It was shown in [7] that these functions can be considered as continuous functions between closure spaces as well as that the corresponding results for function spaces hold in closure spaces, too. In $[9,10,11]$ hyperspaces of closure spaces were introduced and some of their properties expressed by means of selection principles were proved generalizing the well-known topological results (see for example [5]). In the present paper we consider families of subset of a closure space equipped with different Vietoris-like topologies comparing the properties of the space and its hyperspaces.

\section{Preliminaries}

First we recall several definitions.

An operator $u: \mathcal{P}(X) \rightarrow \mathcal{P}(X)$ defined on the power set $\mathcal{P}(X)$ of a set $X$ satisfying the axioms:

2010 Mathematics Subject Classifications. 54A05, 54B20, 54D05, 54D10, 54D30.

Key words and Phrases. Čech closure space, hyperspace, (generalized) Vietoris topology, strong compactness, topological modification.

Received: May 9, 2010

Communicated by Ljubiša Kočinac

* The third author was supported by the Ministry of Science, Technology and Development, Republic of Serbia. 
(C1) $u(\emptyset)=\emptyset$,

(C2) $A \subset u(A)$ for every $A \subset X$,

(C3) $u(A \cup B)=u(A) \cup u(B)$ for all $A, B \subset X$,

is called a Čech closure operator and the pair $(X, u)$ is a Čech closure space. For short, $(X, u)$ will be denoted by $X$ as well, and called a closure space or a space.

A subset $A$ is closed in $(X, u)$ if $u(A)=A$ holds. It is open if its complement is closed.

The interior operator int $t_{u}: \mathcal{P}(X) \rightarrow \mathcal{P}(X)$ is defined by means of the closure operator in the usual way: int $t_{u}=\mathrm{c} \circ u \circ \mathrm{c}$, where $\mathrm{c}: \mathcal{P}(X) \rightarrow \mathcal{P}(X)$ is the complement operator. A subset $U$ is a neighbourhood of a point $x$ in $X$ if $x \in$ $\mathrm{int}_{u} U$ holds.

In $(X, u)$, a point $x \in u(A)$ if and only if for each neighbourhood $U$ of $x, U \cap$ $A \neq \emptyset$ holds.

Separation axioms are defined in the usual way (see [1], Section 27). A space $(X, u)$ is:

$T_{0}$ if for each two distinct points in $X$ at least one has a neighbourhood which does not contain the other point.

$T_{1}$ if for each two distinct points in $X$ the following holds: $(\{x\} \cap u(\{y\})) \cup(\{y\} \cap$ $u(\{x\}))=\emptyset$ whenever $x \neq y$. It is equivalent to: every one-point subset of $X$ is closed in $(X, u)$.

$T_{2}$ (Hausdorff) if each two distinct points have disjoint neighbourhoods.

Regular if for each $x \notin u(A)$ there exist disjoint neighbourhoods $U$ of $x$ and $V$ of $A$. It is equivalent to: for every point $x$ and its neighbourhood $U$ there is a neighbourhood $V$ of $x$ such that $x \in V \subset u(V) \subset U$ holds.

Since every completely regular (Tikhonov) Čech closure space is topological, we consider only spaces with lower separation axioms.

A collection $\left\{G_{\alpha}\right\}$ is an interior cover of a set $A$ in $(X, u)$ if the collection $\left\{\mathrm{int}_{u} G_{\alpha}\right\}$ covers $A$. We suppose that the interior of every element of an interior cover is nonempty.

A subset $A$ in a space $(X, u)$ is compact (respectively countably compact) if every interior cover (respectively countable interior cover) of $A$ has a finite subcover, not necessarily interior.

The following notations are used:

$\mathcal{H}=\{u(A) \mid A \subset X\}, \mathcal{H}^{*}=\mathcal{H} \backslash\{\emptyset\}, \mathcal{J}=\mathcal{H}^{\mathrm{c}}=\left\{\operatorname{int}_{u}(A) \mid A \subset X\right\}$,

$\mathbf{F}(X)$ is the family of all nonempty finite subsets of $X$,

$\mathbf{F}_{n}(X)$ is the family of all nonempty subsets of $X$ that have at most $n$ elements,

$\mathbf{K}(X)$ is the family of all nonempty compact subsets of $X$,

$\mathbf{2}^{X}$ is the family of all nonempty closed subsets of $X$,

$\mathbf{A}(X)$ is the family of all nonempty subsets of $X$. 
The topological modification $\hat{u}$ of the operator $u$ is the finest Kuratowski closure operator coarser than $u$. The corresponding topology $\mathcal{T}(\hat{u})$ consists of all open sets in $(X, u)$.

We also consider the topology $\mathcal{T}(\tilde{u})$ on $(X, u)$ having for a basis the collection $\mathcal{J}$. Its (Kuratowski) closure operator will be denoted by $\tilde{u}$. The collection $\mathcal{H}$ is a base for closed subsets in $(X, \mathcal{T}(\tilde{u}))$.

In general $\hat{u}$ is coarser and $\tilde{u}$ is finer than $u$. Namely, for every $A \subset X, \tilde{u}(A) \subset$ $u(A) \subset \hat{u}(A)$ holds.

Indeed, $x \in \tilde{u}(A)$ if and only if for each $U \subset X, x \in \operatorname{int}_{u} U$ implies $\operatorname{int}_{u} U \cap A \neq \emptyset$, hence $U \cap A \neq \emptyset$, which is equivalent to $x \in u(A)$. It follows next that for every $U=\operatorname{int}_{u} U, x \in U$ implies $U \cap A \neq \emptyset$ which is equivalent to $x \in \hat{u}(A)$.

Note that $u, \hat{u}$ and $\tilde{u}$ coincide when $u$ is a Kuratowski closure operator.

A subset $S$ of a closure space $(X, u)$ is connected ([1], Definition 20 B.1.) if $S=A \cup B$ and $u(A) \cap B=\emptyset=A \cap u(B)$ implies $A=\emptyset$ or $B=\emptyset$.

By Theorem 20. B.2. of [1], a closure space $(X, u)$ is connected if and only if it is not the union of two disjoint nonempty open (open-and-closed) subsets. Hence

Lemma 1. $(X, u)$ is connected if and only if $(X, \hat{u})$ is connected.

Recall that a closure space $(X, u)$ is locally connected at a point $x$ if connected neighbourhoods of $x$ form a local base at $x$ and $(X, u)$ is feebly locally connected at $x \in X$ if there exists a connected neighbourhood of $x$. (See [1], Definition 21 A.1. and Section 21 B.)

Lemma 2. (See [1], 21 B.9.) If a closure space is (feebly) locally connected, then its topological modification possesses the corresponding property.

We introduce the following

Definition A space $(X, u)$ (respectively a subset $A$ ) is strongly compact if every interior cover of $X$ (respectively $A$ ) has a finite interior subcover. In other words, $X$ (respectively $A$ ) is strongly compact in $(X, u)$ if and only if it is $\mathcal{T}(\tilde{u})$-compact.

For a subset $A \subset X$ the usual notations are $A^{+}=\{H \in \mathcal{H} \mid H \subset A\}$ and $A^{-}=\{H \in \mathcal{H} \mid H \cap A \neq \emptyset\}$.

Open sets in $(X, u)$ define the Vietoris topology $\mathbf{V}$ on $\mathbf{A}(X)$ and its subcollections, while the elements of $\mathcal{T}(\tilde{u})$ define the Vietoris topology that will be denoted by $\mathbf{V}^{\#}$.

In the setting of Čech closure spaces the generalized upper and lower Vietoris topologies $\mathbf{W}^{+}$and $\mathbf{W}^{-}$on the family $\mathcal{H}^{*}$ were introduced in [11] in the following way. 
The collection $\mathcal{J}^{+}=\left\{G^{+} \mid G \in \mathcal{J}\right\}$ is a basis for the topology $\mathbf{W}^{+}$on $\mathcal{H}^{*}$, while $\mathbf{W}^{-}$is defined by the collection $\mathcal{J}^{-}=\left\{G^{-} \mid G \in \mathcal{J}\right\}$. Its basis elements are of the form $G_{1}^{-} \cap \cdots \cap G_{n}^{-}$where $G_{i} \in \mathcal{J}$ for $1 \leqslant i \leqslant n, n \in \mathbb{N}$.

We introduce the generalized Vietoris topology on $\mathcal{H}^{*}$ (and in the same way on $\mathbf{A}(X))$ as $\mathbf{W}=\mathbf{W}^{+} \vee \mathbf{W}^{-}$. Its basis elements are of the form

$G^{+} \cap\left(\cap_{i=1}^{n} G_{i}^{-}\right)$where $G, G_{1}, \ldots, G_{n} \in \mathcal{J}$,

for which we use the notation

$<G ; G_{1}, \ldots, G_{n}>=\left\{H \in \mathcal{H}^{*} \mid H \subset G\right.$ and $H \cap G_{i} \neq \emptyset$ for $\left.i=1, \ldots, n\right\}$.

Without loss of generality we suppose that $G_{i} \subset G$ for $i=1, \ldots, n$.

On $\mathbf{A}(X)$ (and its subcollection $\mathcal{H}^{*}$ ) the following holds

$\mathbf{V} \subset \mathbf{W} \subset \mathbf{V}^{\#}$.

When $(X, u)$ is a topological space $\mathbf{V}=\mathbf{W}=\mathbf{V}^{\#}$ all definitions considered in this paper coincide with the corresponding topological ones.

Example 1. Let $(X, \mathcal{T})$ be a topological space and $u=\operatorname{cl}_{\theta}$ be the $\theta$-closure operator in $(X, \mathcal{T}) .\left(x \in \operatorname{cl}_{\theta}(A)\right.$ if each closed neighbourhood of $x$ intersects $\left.A.\right)$ $\theta$-open sets $(X, \mathcal{T})$ form the topology $\mathcal{T}(\tilde{u})$, while the semi-regularization topology of $\mathcal{T}$, whose basis is the family of regular open sets in $(X, \mathcal{T})$, is the topology $\mathcal{T}(\tilde{u})$. ( $\operatorname{cl}_{\theta}$ is a Kuratowski closure operator if and only if $(X, \mathcal{T})$ is a regular space.) By [8], Corollary 1, Proposition 5 and Theorem 2, connectedness and weak local connectedness are shared by the spaces $\left(X, \mathcal{T}_{s}\right),\left(X, \mathrm{cl}_{\theta}\right)$ and $\left(X, \mathcal{T}_{\theta}\right)$, while local connectedness of $\left(X, \mathcal{T}_{s}\right)$ implies that of $\left(X, \mathrm{cl}_{\theta}\right)$.

In particular, let $(X, \mathcal{T})$ be the digital line and $(X, u)$ its $\theta$-closure space $\left(\mathbb{Z}, \operatorname{cl}_{\theta}\right)$. (See [8], Example 4.) A basis for the topology $\mathcal{T}$ is $\mathcal{B}=\{\{2 m-1\} \mid m \in$ $\mathbb{Z}\} \cup\{\{2 m-1,2 m, 2 m+1\} \mid m \in \mathbb{Z}\}$. Since every basis element is regularly open, $\mathcal{T}_{s}=\mathcal{T}$. The only $\theta$-open sets are the empty set and $\mathbb{Z}$, so the space $\left(\mathbb{Z}, \mathcal{T}_{\theta}\right)$ is indiscrete. Thus the spaces $(X, u)$ and $(X, \tilde{u})$ are not (strongly) compact, but they are locally (strongly) compact, connected and locally connected. The space $(X, \hat{u})$ satisfies all the listed properties.

In general, connectedness is not shared by $(X, u)$ and $(X, \tilde{u})$. It can be seen from the next simple example.

Example 2. Let $X=\{a, b, c\}, u(\emptyset)=\emptyset, u(\{a\})=\{a, b\}, u(\{b\})=\{b, c\}$, $u(\{c\})=\{c, a\}, u(\{a, b\})=u(\{a, c\})=u(\{b, c\})=u(X)=X$. Then $\operatorname{int}_{u} \emptyset=$ $\operatorname{int}_{u}\{a\}=\operatorname{int}_{u}\{b\}=\operatorname{int}_{u}\{c\}=\emptyset, \operatorname{int}_{u}\{a, b\}=\{b\}, \operatorname{int}_{u}\{b, c\}=\{c\}, \operatorname{int}_{u}\{a, c\}=$ $\{a\}$, int $_{u} X=X$. The space $(X, \hat{u})$ is indiscrete while $(X, \tilde{u})$ is discrete. The induced topologies $\mathbf{V}$ and $\mathbf{V}^{\#}$ are indiscrete and discrete respectively.

$\mathcal{H}^{*}=\{\{a, b\},\{a, c\},\{b, c\}, X\}$ and its generalized Vietoris topology

$\mathbf{W}=\{\emptyset,\{X\},\{X,\{a, b\}\},\{X,\{a, c\}\},\{X,\{b, c\}\},\{X,\{a, b\},\{a, c\}\}$,

$\left.\{X,\{a, b\},\{b, c\}\},\{X,\{a, c\}\{b, c\}\}, \mathcal{H}^{*}\right\}$. Thus $\left(\mathcal{H}^{*}, \mathbf{W}\right)$ is (hyper)connected, while $(\mathbf{A}(X), \mathbf{W})$ is disconnected since $\{a\}^{-} \cup\{b\}^{-}=\{\{a\},\{b\},\{a, b\},\{a, c\},\{b, c\}, X\}$ 
and $\{c\}^{+}=\{\{c\}\}$ form a decomposition of $\mathbf{A}(X)$.

All notions not explained here concerning Čech closure spaces can be found in [1] and [7].

\section{Some properties of the hyperspaces of $(X, u)$}

In the sequel all considered spaces are $\mathrm{T}_{1}$.

By $\mathbf{C}(X)$ we denote the family of all nonempty compact subsets in $(X, \hat{u})$, and by $\mathbf{Q}(X)$ the family of all nonempty closed subsets in $(X, \mathcal{T}(\tilde{u}))$.

Lemma 3. For a space $(X, u)$ the following hold:

(i) $A^{+}$and $A^{-}$are closed sets for every $A \in \mathcal{H}$.

(ii) $\mathrm{cl}<G ; G_{1}, \ldots, G_{n}>\subset<u(G) ; u\left(G_{1}\right), \ldots, u\left(G_{n}\right)>$.

(iii) If $\left\{U_{\lambda}\right\}$ is a neighbourhood basis of $x \in X$, then $\left\{\left(\operatorname{int}_{u} U_{\lambda}\right)^{+}\right\}$is a neighbourhood basis of $\{x\} \in \mathcal{H}^{*}$.

Proof. (i) The statement follows from the equalities: $\left(A^{+}\right)^{c} \equiv \mathcal{H}^{*} \backslash A^{+}=\left(A^{c}\right)^{-}$ and $\left(A^{-}\right)^{c} \equiv \mathcal{H}^{*} \backslash A^{-}=\left(A^{c}\right)^{+}$.

(ii) Since $u(G), u\left(G_{1}\right), \ldots, u\left(G_{n}\right) \in \mathcal{H}^{*}$, the collections $(u(G))^{+},\left(u\left(G_{1}\right)\right)^{+}, \ldots,\left(u\left(G_{n}\right)\right)^{+}$ are closed in $\left(\mathcal{H}^{*}, \mathbf{W}\right)$. Thus, $<u(G) ; u\left(G_{1}\right), \ldots, u\left(G_{n}\right)>$ is closed and $\mathrm{cl}<$ $G ; G_{1}, \ldots, G_{n}>\subset<u(G) ; u\left(G_{1}\right), \ldots, u\left(G_{n}\right)>$.

For the converse, let $H \in\left\langle u(G) ; u\left(G_{1}\right), \ldots, u\left(G_{n}\right)\right\rangle$ and $\left\langle U ; U_{1}, \ldots, U_{m}\right\rangle$ be a neighbourhood of $H$, where $U=\operatorname{int}_{u} A$ and $U_{j}=\operatorname{int}_{u} A_{j}, j=1, \ldots, m$. Then $H \subset U, H \cap U_{j} \neq \emptyset$ for $j=1, \ldots, m ; H \subset u(G)$ and $H \cap u\left(G_{i}\right) \neq \emptyset$ for $i=$ $1, \ldots, n$. There is $x_{i} \in H \cap u\left(G_{i}\right)$ for each $i \in\{1, \ldots, n\}$, and for the neighbourhood $A$ of $x_{i}$, there is a $z_{i} \in A \cap G_{i}$. In a similar way, for each $j \in\{1, \ldots, m\}$, there is $y_{j} \in H \cap U_{j}$ and for the neighbourhood $A_{j}$ of $y_{j}$, there is a $\hat{z}_{j} \in A_{j} \cap G$. The set $K=\left\{z_{i} \mid i=1, \ldots, n\right\} \cup\left\{\hat{z}_{j} \mid j=1, \ldots, m\right\} \in<A ; A_{1}, \ldots, A_{m}>\cap<$ $G ; G_{1}, \ldots, G_{n}>=<A \cap G ; A_{1} \cap G, \ldots, A_{m} \cap G, G_{1} \cap A, \ldots, G_{n}, \cap A>$.

In fact, $\mathrm{cl}<C ; C_{1}, \ldots, C_{n}>\subset<u(C) ; u\left(C_{1}\right), \ldots, u\left(C_{n}\right)>$ holds for any nonempty sets $C, C_{1}, \ldots, C_{n}, C_{i} \subset C$.

(iii) Clear. $x \in \operatorname{int}_{u} U_{\lambda} \Leftrightarrow\{x\} \in\left(\operatorname{int}_{u} U_{\lambda}\right)^{+}$holds.

Proposition 1. For a space $(X, u)$ the following hold:

(i) $\mathbf{F}(X)$ is dense in $\left(\mathcal{H}^{*}, \mathbf{W}\right)$.

(ii) If $(X, u)$ is $T_{2}$, then $\mathbf{F}_{n}(X)$ is closed in $\left(\mathcal{H}^{*}, \mathbf{W}\right)$ for all $n \geqslant 1$.

(iii) The natural projection $p:(X, u)^{n} \rightarrow\left(\mathbf{F}_{n}(X), \mathbf{V}\right)$ defined by $p\left(x_{1}, \ldots, x_{n}\right)=\left\{x_{1}, \ldots, x_{n}\right\}$, is continuous.

Proof. (i) For each nonempty open set $\left\langle G ; G_{1}, \ldots, G_{n}>\right.$, let $x_{i} \in G_{i}, i=$ $1, \ldots, n$. The set $\left\{x_{1}, \ldots, x_{n}\right\}$ is finite and belongs to $\left\langle G ; G_{1}, \ldots, G_{n}\right\rangle$. 
(ii) For a fixed $n \in \mathbb{N}$, let $H \in \mathcal{H}^{*} \backslash \mathbf{F}_{n}$ and $x_{1}, \ldots, x_{n+1}$ be distinct points in $H$. Since the space $(X, u)$ is $\mathrm{T}_{2}$, let $U_{1}, \ldots, U_{n+1}$ be pairwise disjoint neighbourhoods of $x_{1}, \ldots, x_{n+1}$, respectively. Let $U$, be a neighbourhood of $H$, and $U_{i} \subset U$, for $i=1, \ldots, n+1$. (It can be chosen $U=X$.) Then $<\operatorname{int}_{u} U ; \operatorname{int}_{u} U_{1}, \ldots, \operatorname{int}_{u} U_{m}>$ is a neighbourhood of $H$, and $<\operatorname{int}_{u} U ; \operatorname{int}_{u} U_{1}, \ldots, \operatorname{int}_{u} U_{m}>\cap \mathbf{F}_{n}=\emptyset$.

(iii) $\left(\mathbf{F}_{n}(X), \mathbf{V}\right)$ is a topological space so the mapping $p:\left(X^{n}, v\right) \rightarrow\left(\mathbf{F}_{n}(X), \mathbf{V}\right)$ is continuous if and only if the mapping $p:\left(X^{n}, \hat{v}\right) \rightarrow\left(\mathbf{F}_{n}(X), \mathbf{V}\right)$ is continuous, where $\hat{v}$ is the topological modification of $v$. Since the topological modification of the product space is the product of topological modifications, $p$ is continuous if and only if it is continuous as the mapping from the $n$th product of the topological space $(X, \mathcal{T}(\hat{u}))$ into $\left(\mathbf{F}_{n}(X), \mathbf{V}\right)$, which follows from [6], Proposition 2.4.3.

Proposition 2. (i) If $A$ is dense in $(X, u)$, then $\mathbf{F}(A)$ and $\mathbf{2}^{A}$ are dense in $(\mathbf{A}(X), \mathbf{V})$. $T_{1}$.

(ii) For a $T_{1}$ space $(X, u)$, the spaces $\left(\mathbf{2}^{X}, \mathbf{V}\right),\left(\mathcal{H}^{*}, \mathbf{W}\right)$ and $\left(\mathbf{Q}(X), \mathbf{V}^{\#}\right)$ are

(iii) If $(X, u)$ is regular, then $\left(\mathbf{2}^{X}, \mathbf{W}\right)$ is $T_{2}$.

Proof. (i) If $A$ is dense in $(X, u)$, then $A$ is dense in $(X, \hat{u})$ and the statement follows from definitions and Corollary 5.2.4 of [6].

(ii) Let $H_{1}, H_{2} \in \mathcal{H}^{*}, H_{1} \neq H_{2}$ and $x_{1} \in H_{1} \backslash H_{2}$. Then $H_{1} \in\left(H_{2}^{c}\right)^{-}=\mathcal{W}_{1}$ holds and $H_{2} \notin \mathcal{W}_{1}$. If there is $x_{2} \in H_{2}$, then $H_{2} \in\left(H_{1}^{c}\right)^{-}=\mathcal{W}_{2}$ and $H_{1} \notin \mathcal{W}_{2}$ holds. If $H_{2} \subset H_{1}$, then $H_{2} \in\left(\left\{x_{1}\right\}^{c}\right)^{+}=\mathcal{W}_{2}$ and $H_{1} \notin \mathcal{W}_{2}$.

(iii) Let $A_{1}, A_{2} \in \mathbf{2}^{X}, A_{1} \neq A_{2}, A_{1}=u\left(A_{1}\right), A_{2}=u\left(A_{2}\right)$ and $x_{1} \in A_{1} \backslash A_{2}$. By regularity of $(X, u)$ there are disjoint neighbourhoods $U$ of $x$ and $V$ of $A_{2}$. Hence $x \in \operatorname{int}_{u}(U)$ and $A_{2} \subset \operatorname{int}_{u}(V)$ imply $A_{1} \in\left(\operatorname{int}_{u}(U)\right)^{-}=\mathcal{W}_{1}, A_{2} \in$ $\left(\operatorname{int}_{u}(V)\right)^{+}=\mathcal{W}_{2}$ and $\mathcal{W}_{1} \cap \mathcal{W}_{2}=\emptyset$.

Proposition 3. A space $(X, u)$ is connected if and only if its nth product $\left(X^{n}, v\right),(n=$ $1,2, \ldots)$, is connected.

Proof. The spaces $(X, u)$ and its product $\left(X^{n}, v\right)$ are connected if and only if their topological modification $(X, \hat{u})$ and $\left(X^{n}, \hat{v}\right)$ are connected. Since $\left(X^{n}, \hat{v}\right)$ is the $n$th product of $(X, \hat{u})$, the statement follows.

Proposition 4. If $\sigma$ is a connected collection in $(\mathbf{A}(X), \mathbf{W})$, and one of whose elements is connected, then $E=\cup\{S \in \sigma\}$ is connected.

Proof. Let $S_{0} \in \sigma$ be a connected subset. If $E=\cup\{S \in \sigma\}$ is not connected, then $E=A \cup B$ where $A$ and $B$ are disjoint, nonempty and open-and-closed. Since $S_{0}$ is connected, $S_{0} \subset A$ or $S_{0} \subset B$ holds. Suppose $S_{0} \subset A$; then $S_{0} \in A^{+}$. It follows that $A^{+}$and $B^{-}$form a decomposition of $\sigma$.

Theorem 1. Let $\mathbf{F}(X) \subset \sigma \subset \mathbf{A}(X)$. 
(i) If one of the spaces $(X, u),\left(\mathbf{F}(X)_{n}, \mathbf{V}\right), n \in \mathbb{N}$, or $(\sigma, \mathbf{V})$ is connected, then all of them are connected.

(ii) Connectedness of one of the spaces $\left(\mathbf{F}(X)_{n}, \mathbf{W}\right), n \in \mathbb{N}$, or $(\sigma, \mathbf{W})$ implies connectedness of $(X, u)$.

Proof. (i) Follows by Lemma 1 and Theorem 4.10 of [6].

(ii) In a similar way, by Lemma 1 , Proposition 4 and the fact that $\mathbf{V} \subset \mathbf{W}$.

Note that connectedness of all $\left(\mathbf{F}(X)_{n}, \mathbf{W}\right), n \in \mathbb{N}$, implies connectedness of $(\mathbf{F}(X), \mathbf{W})$ and hence of $(\sigma, \mathbf{W})$.

Proposition 5. (Feeble) local connectedness of $(X, u)$ implies (feeble) local connectedness of $(\sigma, \mathbf{V})$, where $\mathbf{F}(X) \subset \sigma \subset \mathbf{C}(X)$.

Proof. By Lemma 2, (feeble) local connectedness of $(X, u)$ implies (feeble) local connectedness of its topological modification $(X, \hat{u})$. Local connectedness of $(\sigma, \mathbf{V})$ follows by applying Theorem 4.12 in [6]. We modify the same proof for feeble local connectedness.

Proposition 6. (i) If $\left(\mathcal{H}^{*}, \mathbf{W}\right)$ is (countably) compact, then $(X, u)$ is strongly (countably) compact.

(ii) The space $\left(\mathbf{2}^{X}, \mathbf{V}\right)$ is compact if and only if $(X, \hat{u})$ is compact.

(iii) The space $\left(\mathbf{Q}(X), \mathbf{V}^{\#}\right)$ is compact if and only if $(X, \tilde{u})$ is compact, that is, $(X, u)$ is strongly compact.

Proof. (i) Let $\left\{G_{\alpha}\right\}$ be a (countable) interior cover of $X$. Then $\cup\left\{\operatorname{int}_{u} G_{\alpha}\right\}=$ $X$. The collection $\left\{\left(\operatorname{int}_{u} G_{\alpha}\right)^{-}\right\}$is a (countable) open cover of $\mathcal{H}^{*}$ and there is a finite subcover $\left\{\left(\operatorname{int}_{u} G_{\alpha_{i}}\right)^{-} \mid i=1, \ldots m\right\}$. Hence $\left\{G_{\alpha_{i}}, \mid i=1, \ldots m\right\}$ is a finite interior cover of $X$, a subcover of $\left\{G_{\alpha}\right\}$.

(ii) and (iii) follow by [6], Theorem 4.2.

The next two statements are a weaker form of [6], Teorem 2.5.

Proposition 7. If $\mathcal{C}$ is a compact collection in $\left(\mathcal{H}^{*}, \mathbf{W}\right)$ consisting of strongly compact elements, then $K=\cup\{C \in \mathcal{C}\}$ is compact.

Proof. Let $\left\{G_{\alpha}\right\}$ be an interior cover of $K$. For each $C \in \mathcal{C}$ there is a finite subcollection $\left\{G_{C, 1}, \ldots, G_{C, n(C)}\right\}$ whose interiors cover $C$. Put $G_{C}=$ $\cup\left\{G_{C, 1}, \ldots, G_{C, n(C)}\right\}$. Then $\left\{<\operatorname{int}_{u} G_{C} ; \operatorname{int}_{u} G_{C, 1}, \ldots\right.$, int $\left._{u} G_{C, n(C)}>\right\}$, where $C \in \mathcal{C}$, is an open cover of $\mathcal{C}$, so there is a finite subcover $\left\{<\operatorname{int}_{u} G_{C_{i}} ;\right.$ int $\left._{u} G_{C_{i}, 1}, \ldots, \operatorname{int}_{u} G_{C_{i}, n\left(C_{i}\right)}>\mid i=1, \ldots, m\right\}$. The collection $\left\{G_{C_{i}}, G_{C_{i}, 1}, \ldots, G_{C_{i}, n\left(C_{i}\right)} \mid i=\right.$ $1, \ldots, m\}$ is a finite cover of $K$, a subcollection of $\left\{G_{\alpha}\right\}$.

Proposition 8. If $(X, u)$ is a regular space and $\mathcal{C}$ is a compact collection in $\left(\mathcal{H}^{*}, \mathbf{W}\right)$ consisting of closed elements, then $K=\cup\{C \in \mathcal{C}\}$ is closed. 
Proof. Let $\mathcal{C}=\left\{A_{\lambda}=u\left(A_{\lambda}\right) \mid \lambda \in \Lambda\right\}$. Let $x \in u(K)$. By regularity, for each neighbourhood $U$ of $x$ there is a neighbourhood $V$ of $x$ such that $x \in$ $V \subset u(V) \subset U$ holds. Then $x \in u(K)$ implies $V \cap K \neq \emptyset$, hence there is a $\lambda \in \Lambda$ such that $u(V) \cap A_{\lambda} \neq \emptyset$, i. e. $A_{\lambda} \in(u(V))^{-}$. By Lemma 3(i) the collection $\mathcal{C} \cap(u(V))^{-}$is closed in $\mathcal{C}$, and $\left\{\mathcal{C} \cap(u(V))^{-} \mid V \in \mathcal{N}(x)\right\}$ has the finite intersection property since $V_{1}, \ldots, V_{n} \in \mathcal{N}(x)$ imply $V_{1} \cap \ldots \cap V_{n} \in \mathcal{N}(x)$. Since $(\mathcal{C}, \mathbf{W})$ is compact, there is a nonempty subcollection $\mathcal{E} \subset \mathcal{C}$. For each $E \in \mathcal{E}, E \in \cap\left\{(u(V))^{-} \mid V \in \mathcal{N}(x)\right\}$ implies $E \cap U \neq \emptyset$ for each $\left.U \in \mathcal{N}(x)\right\}$, hence $x \in u(E)=E$. Since $E \subset K, x \in K$. Hence $u(K)=K$.

\section{References}

[1] E. Čech, Topological Spaces, Revised edition by Z. Frolík and M. Katětov, Czechoslovak Acad. of Sciences, Prague, 1966.

[2] D.N. Georgiou, A few remarks concerning $\vartheta$-continuous functions and topologies on function spaces, J. Inst. Math. Comput. Sci. (Math. Ser.)12 (1999), $129-138$.

[3] D.N. Georgiou and B.K. Papadopoulos, Strongly $\theta$-continuous functions and topologies on function spaces, Appl. Categ. Structures, 2000, 433-444.

[4] D.N. Georgiou and B.K. Papadopoulos, Weakly continuous, weakly $\theta$ continuous, super-continuous and topologies on function spaces, Sci. Math. $\mathbf{5 3}$ (2001).

[5] Lj.D.R. Kočinac, Selection principles in hyperspaces, in: Lj.D.R. Kočinac (Ed.), Selection Principles and Covering Properties in Topology, Quad. Mat. 18 (2006), 157-194.

[6] E. Michael, Topologies on spaces of subsets, Trans. Amer. Math. Soc. 71 (1951), $152-182$.

[7] M. Mršević, Proper and admissible topologies in closure spaces, Indian J. Pure Appl. Math. 36 (2005), 613-627.

[8] M. Mršević and D. Andrijević, On $\theta$-conectedness and $\theta$-closure spaces, Topology Appl. 123 (2002), 157-166.

[9] M. Mršević and M. Jelić, Selection principles and hyperspace topologies in closure spaces, J. Korean Math. Soc. 43 (2006), 1099-1114.

[10] M. Mršević and M. Jelić, Selection principles, $\gamma$-sets and $\alpha_{i}$-properties in Čech closure spaces, Topology Appl. 155 (2008), 1947-1958.

[11] M. Mršević and M. Jelić, Selection principles in hyperspaces with generalized Vietoris topologies, Topology Appl. 156 (2008), 124-129. 


\section{Addresses:}

Dimitrije Andrijević

Poljoprivredni fakultet, University of Belgrade, Nemanjina 6, 11081 Beograd-Zemun, Serbia

E-mail: adimitri@agrif.bg.ac.rs

Milena Jelić

Poljoprivredni fakultet, University of Belgrade, Nemanjina 6, 11081 Beograd-Zemun, Serbia

E-mail: milena@agrif.bg.ac.rs

Mila Mršević

Faculty of Mathematics, University of Belgrade, Studentski trg 16, 11000 Beograd, Serbia

E-mail: mrsevic@matf.bg.ac.rs 